\title{
Experimental Evidence of Kink Relaxation and Kink Resonance in Dislocations of FCC Metals
}

\author{
A.O. Moreno-Gobbi, J.A. Eiras*, M.A. Marotti and A. Ghilarducci** \\ Inst. de Física, Fac. de Ciencias, Tristán Narvaja 1674, CP 11200, Montevideo, Uruguay \\ * Centro Atómico CC 439, 8400 Bariloche RN, Argentina \\ ** Dept. Física, UFSCar-Rod, Washington Luiz km 235, CEP 13565-905, São Carlos SP, Brazil
}

\begin{abstract}
Many papers have been devoted to the motion of dislocation lines in crystals. The string model and the kink model presented the most relevant theoretical approach. However, each model independently can not describe all experimental data. In this paper is presented experimental data of logarithmic decrement associated to dislocation lines, measured in $\mathrm{Hz}$ and $\mathrm{MHz}$ ranges, on high purity copper single crystals plastically deformed by compression and torsion at room temperature. The experimental data obtained for the Bordoni Peak, analyzed on the basis of the kink model, shows the predicted temperature dependence of the effective enthalpy, a evidence of the kink diffusion mechanism. The data can be well fitted over all temperature range investigated, assuming the contribution of kink relaxation and kink resonance aging simultaneously. From the last mechanism a temperature dependent kink density is assumed. This procedure allows to explain the width and the asymmetry of the Bordoni peak.
\end{abstract}

\section{INTRODUCTION}

The high asymmetry and the great width of the Bordoni Peak (BP) [1] compared to a Debye one are two relevant characteristics of the BP. Moreno-Gobbi and Eiras [2] proposed that the asymmetry of the BP may be due to the contributions of two different dislocation mechanisms: kink relaxation (with a Debye realation time) and overdamped kink resonance. But the width of the fitted peaks remains lower than the experimental peaks.

In this paper we propose that the large width of the high frequency BPs may be understood considering the relaxation time calculated by Seeger [4] using the diffusion theory of kink-pair formation in dislocations. The most relevant theoretical characteristic of this mechanism is the existence of an effective kink pair creation enthalpy which is temperature dependent.

This paper presents an Arrhenius plot with data obtained from BPs measurements at $\mathrm{Hz}$ and $\mathrm{MHz}$ frequencies, on samples cut from the same original crystal and also submitted to identical thermal treatment. Completing the frequency range, all other measurements on copper samples reported in the literature were included to complete the frequency range.

The interpretation of high frequency $(10$ to $50 \mathrm{MHz})$ logarithmic decrement obtained from ultrasonic attenuation measured on a sample of high purity crystalline copper plastically deformed in $5 \%$ at room temperature, was done in terms of a superposition of kink relaxation and kink resonance were done, considering Seeger's model of creation of kink-pairs submitted to kink-diffusion, and kink-resonance with a temperature dependent kink density.

\section{THEORETICAL CONSIDERATIONS}

In Seeger's model [4] the Bordoni relaxation is due to the thermally activated formation of kink-pairs submitted to diffusion, with the relaxation time

$$
\tau=\frac{k_{B} L}{\pi w_{k} H_{k}} \frac{T}{D_{k}} \frac{\left(1+\rho_{k}^{e q} L / 2\right)}{\left(\rho_{k}^{e q}\right)^{2}}
$$


where $L=$ average free length of segment of the dislocation line; $w_{k}=$ kink width; $H_{k}=$ half the formation activation enthalpy of kink-pairs; $D_{k}=$ kink diffusion coefficient and $\rho_{k} e q=$ average density of kinks in thermal equilibrium on the dislocation. For temperatures above the value $\theta_{\mathrm{D}} 2 \mathrm{a} / \pi \mathrm{w}_{\mathrm{K}}=44 \mathrm{~K}\left(\theta_{\mathrm{D}}=343 \mathrm{~K}=\right.$ Debye temperature of copper; $\mathrm{a}=$ interatomic distance in the dislocation line), it can be applied the high temperature approximation for $\rho_{\mathrm{k}}{ }^{\mathrm{eq}}$

$$
\rho_{\mathrm{k}}^{\text {eq,ht }}=\frac{1}{w_{k}}\left(\frac{2 \pi H_{k}}{2 k_{B} T}\right)^{1 / 2} \exp \left(\frac{-H_{k}}{k_{B} T}\right)
$$

A relevant consequence of this relaxation time is the existence of an equivalent creation enthalpy $\mathrm{H}_{\mathrm{eff}}$ which is temperature dependent

$$
H_{s f f}=\frac{d \ln \tau}{d\left(1 k_{B} T\right)}
$$

At measurements with high frequency ultrasonic waves, the Bordoni peak will be in the high temperature region (120 to $170 \mathrm{~K})$. As a consequence of eqs (1-2), at high temperatures $(\rho \mathrm{L} / 2 \gg 1)$ the relaxation time is

$$
\tau=\left(\mathrm{L}^{2} \mathrm{w}_{\mathrm{k}} / 4 \mathrm{a}^{2} \mathrm{~S}_{\mathrm{d}} \mathrm{D}_{\mathrm{k}}\right)\left(\mathrm{k}_{\mathrm{B}} \mathrm{T}\right)^{3 / 2} \exp \left(\mathrm{H}_{\mathrm{k}} / \mathrm{k}_{\mathrm{B}} \mathrm{T}\right)
$$

This expresion for $\tau$ means that at high frequencies the BP relaxation becomes wider than a Debye peak (DP). This fact is very important considering that at high frequencies the BPs are widened in about 2.5 times a DP [3].

On dislocation lines at little angles with Peierls valleys geometrical kinks must be present. Suzuki and Elbaum [5] proposed that resonance of these kink chain may occur. Alefeld [6] pointed out that those chains may have a density of kinks which is a function of temperature. Above certain critical temperature $T_{C}$ ( $\cong 150 \mathrm{~K}$ for copper, considering 15 geometrical kinks in the dislocation line) the thermal kinks will also be created with a zero applied stress, contributing to the resonance component with a temperature dependent density. Moreno-Gobbi and Eiras showed that the addition of the resonance mechanism to relaxation allows to explain the large asymmetry of the BP [2].

For the simultaneous contributions of relaxation and resonance mechanisms [2], the general expression for the logarithmic decrement is

$$
\begin{aligned}
\delta= & \Delta_{\mathrm{B}} \omega \tau /\left[1+(\omega \tau)^{2}\right]+\mathrm{A}_{1} \mathrm{~N}_{\mathrm{k}}(\mathrm{T}) \mathrm{B}(\mathrm{T}) \omega+\delta_{\mathrm{o}}= \\
= & \Delta_{\mathrm{B}} \omega \tau /\left[1+(\omega \tau)^{2}\right]+\mathrm{A}_{2} \mathrm{~T}^{2}+\delta_{\mathrm{o}} \\
& \tau=\mathrm{A}_{2}\left(\mathrm{k}_{\mathrm{B}} \mathrm{T}\right)^{3 / 2} \exp \left(\mathrm{H}_{\mathrm{k}} / \mathrm{k}_{\mathrm{B}} \mathrm{T}\right)
\end{aligned}
$$

where $\Delta_{B}=$ Bordoni relaxation intensity; $A_{1}$ and $A_{2}=$ constants which depend on the material and the dislocation structure, $N_{k}(T)=$ density of kinks participating in the resonance process, $\omega=$ wave angular frequency and $\delta_{0}=$ constant. For the ressonance component an overdamped process is considered, were $\mathrm{N}_{k}(T) \propto T[2]$ and $B(T) \propto T$ [7], and the low frequency approximation $\left(\omega<<\omega_{\text {es }}\right.$, reonance frequency) is used [2]. 


\section{EXPERIMENTAL DATA AND DISCUSSION}

Attenuation measurements were made by the pulse-echo method [8], cooling the sample between $5 \mathrm{~K}$ and $373 \mathrm{~K}$ at a maximum rate of one degree per minute, on a high purity crystalline copper sample deformed plastically in 5\% in the [111] direction. Measurements were obtained at 10, 30 and $50 \mathrm{MHz}$.

For low frequency internal friction measurements, a sample of $19.55 \times 2.45 \times 0.90 \mathrm{~mm}$ cut from the same crystal of the ultrasonic samples was used. Also this sample was prepared in an identical way as the ultrasonic samples. Measurements at 5 to $20 \mathrm{~Hz}$ were obtained with a subresonant torsion pendulum and are reported in another paper [9].

In fig. 1 an Arrhenius plot with all copper temperature peaks obtained from the literature $[3,7]$ and those obtained from our samples is shown. In this plot the fitted curve with the model of eqs. (1) and (2) is shown, finding a very good agreement with experimental data. From the parameters of the fitted curve we obtained $\mathrm{D}_{\mathrm{k}} \approx 2,91 \mathrm{~cm}^{2} / \mathrm{s}, \mathrm{w}_{\mathrm{k}} / \mathrm{L} \approx 2.10^{-3}$ and $\mathrm{H}_{\mathrm{k}} \approx 0.075 \mathrm{eV}$. The value of $\mathrm{D}_{\mathrm{k}}$ is not yet reported, but the other data are in very good agreement with the expected data from the literature. This fact contributes to the confirmation of the diffusion process controlling kink mobility, a fact which do not have yet an experimental confirmation [10].

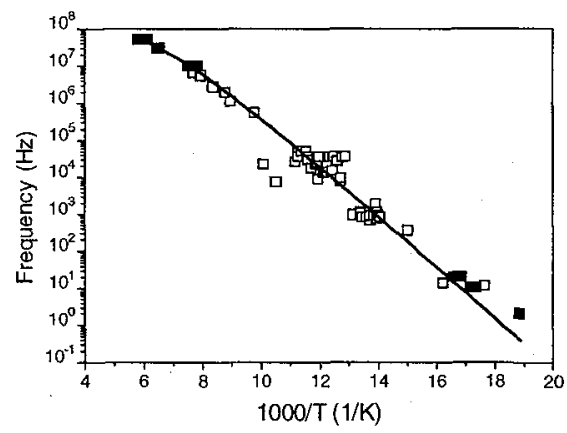

Fig. 1. Dots: Arrhenius plot for data measured on samples cut from the same original copper crystal, in the $\mathrm{Hz}$ and $\mathrm{MHz}$ range. Experimental data of copper from other authors are also included, specially at intermediate frequencies. Full line: fitted curve using equations (2) and (3), with $\mathrm{H}_{\mathrm{k}}=0.074 \mathrm{eV}, \mathrm{L}=10^{4} \mathrm{a}, \mathrm{w}_{\mathrm{k}}=20 \mathrm{a}$ and $\mathrm{a}=5$. $10^{-8} \mathrm{~cm}$.

In figures 2(a) and 2(b) experimental data (dots) of logarithmic decrement in the temperature range from 373 to $80 \mathrm{~K}$, measured at 30 and $50 \mathrm{MHz}$ in a $5 \%$ deformed sample are shown. The line curves are the fitted curves obtained with the model proposed for $\delta$, equations (5) and (6). Because the second BP modifies the $\delta$-curves below $80 \mathrm{~K}$, this region was not considered in the fits and were omitted in the figures. The coefficients obtained from the Arrhenius fit were employed in the logarithmic decrement fitting equation, and a relaxation strength of $2.1 \times 10^{-3}$ was obtained from the fitted curves.

The good agreement obtained in fitting the experimental data shows that they can be interpreted in terms of the two mechanisms proposed. The overdamped resonance mechanism is very important in the interpretation of modulus defect at temperatures above peak temperature, as Moreno-Gobbi and Eiras recently pointed out [2] and also it results very important in the interpretation of the high temperature side of the BP. The Seeger's theory with kink diffusion is particularly interesting to explain the great width of the Bordoni peak at $\mathrm{MHz}$ frequencies. 


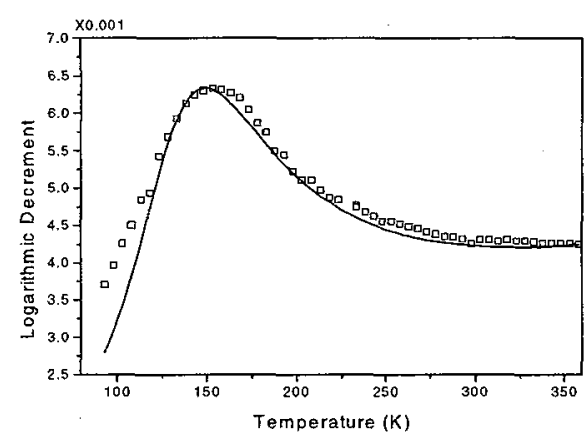

Fig.2(a) Dots: Logarithmic decrement measured at $30 \mathrm{MHz}$ on a high purity crystalline copper sample plastically deformed at room temperature in $5 \%$. Line: fitted curve obtained with the contribution of relaxation and kink-resonance mechanisms, equations (5) and (6).

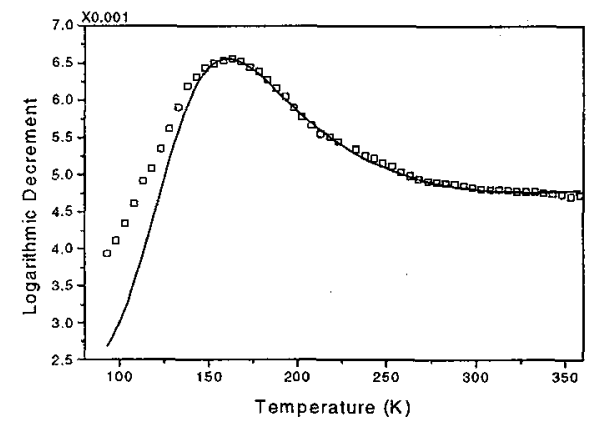

Fig.2(b) Dots: Logarithmic decrement measured at $50 \mathrm{MHz}$ on the same sample of fig. 2(a). Line: fitted curve obtained with the contribution of relaxation and kink-resonance mechanisms, equations (5) and (6).

\section{CONCLUSIONS}

The logarithmic decrement in crystalline copper samples was investigated at Mhz frequencies. The samples were oriented in the $<111\rangle$ crystallographic direction and plastically deformed in $5 \%$. The data were fitted considering the simultaneous contributions of two different dislocation mechanisms: overdamped kink-resonance and kink-relaxation. A linear temperature dependendence of kink density was assumed for the kink resonance in the temperature range considered. The Bordoni relaxation was described considering the kink pairs creation process submitted to a diffusion process. An effective activation enthalpy of $0.075 \mathrm{eV}$ was obtained from an Arrhenius plot fitted with the same relaxation model. Also the relaxation intensity and the diffusion coefficient were obtained, being $2.1 \times 10^{-3}$ and $2.91 \mathrm{~cm}^{2} / \mathrm{s}$ respectively. The fitted curves shows that the diffusion mechanism proposed in the kink-pair creation process is experimentally supported. Also an overdamped resonance mechanism is necessary in order to fit the high temperature side of the BPs, especially those measured at high frequencies.

\section{References}

[1] Bordoni, P. G.,8 Ric. Sci., 19 (1949) 851.

[2] Moreno-Gobbi, A. and Eiras, J. A., Journal Alloys and Compounds, 211-212 (1994) 152.

[3] Moreno-Gobbi, A. and Eiras, J.A., Mater. Sci. Forum, 119-120 (1993) 189

[4] Seeger, A., J. Physique, 42 (1981) C5-201.

[5] Suzuki; T.;. Elbaum, C., J. Appl. Phys., 35 (1964) 1539.

[6] Alefeld, G., Chambers, R. H. and Firle, T. E., J. Appl. Phys., 36 (1965) 2642.

[7] Niblett, D. H., Bordoni Peak in Face Centered Cubic Metals, in Physical Acoustics, ed. by W. P.Mason Vol. IIIA, Part A, Academic Press, New York, 1966.

[8] Papadakis, E.P., in Physical Acoustics, de. by R.N.Thurston and A.D.Pierce, Vol. XIX, Academic Press, New York, 1990.

[9] Ghilarducci, A., Moreno-Gobbi, A., Marotti, M. and Eiras, J. A. Proceedings from 11 ICIFUAS, France, 1996.

[10] Richie, Y., Fantozzi, G., Internal Friction due to the Intrinsic Propierties of Dislocations in Metals: Kink Relaxation, in Dislocations in Solids, ed. by F.R.N. Nabarro, chap.45, Elseiver Sci. Publ., 1992. 